\title{
Proto-Oncogene Tyrosine-Protein Kinase Receptor Ret
}

National Cancer Institute

\section{Source}

National Cancer Institute. Proto-Oncogene Tyrosine-Protein Kinase Receptor Ret. NCI

Thesaurus. Code C18539.

Proto-oncogene tyrosine-protein kinase receptor Ret (1114 aa, 124 kDa) is encoded by the human RET gene. This protein plays a role in the proliferation, migration and differentiation of neural cells. 